\title{
Single-Nucleotide Polymorphisms in the p53 Pathway
}

\author{
S.L. HARris, ${ }^{* \dagger}$ G. GIL, ${ }^{* \dagger}$ W. Hu, ${ }^{*}$ H. Robins,${ }^{\ddagger}$ E. Bond, ${ }^{*}$ K. Hirshrield ${ }^{*}$ Z. FenG, ${ }^{*}$ X. Yu, ${ }^{*}$ \\ A.K. TERESKY, * G. BOND, AND A.J. LEVINE* ${ }^{* \neq}$ \\ *The Cancer Institute of New Jersey, Robert Wood Johnson Medical School, New Brunswick, New Jersey 08903; \\ Institute for Advanced Study, School of Natural Sciences, Princeton, New Jersey 08540
}

\begin{abstract}
A cell culture assay has been developed that detects and validates single-nucleotide polymorphisms (SNPs) in genes that populate the p53 pathway. One hundred thirteen EBV-transformed human B-lymphocyte cell lines obtained from a diverse population were employed to measure the apoptotic response to gamma radiation. Each cell line undergoes a reproducible, characteristic frequency of apoptosis, and the response of the population forms a normal distribution around a median of $35.5 \%$ apoptosis with a range from $12 \%$ to $58 \%$ apoptosis. Polymorphisms in the AKT1 and Perp genes significantly affect the frequency of apoptosis. The assay can detect both racial and sexual dimorphisms in these genes and has the ability to demonstrate epistatic relationships within the p53 pathway. The cell lines used in this assay provide biological materials to explore the molecular basis of the polymorphisms.
\end{abstract}

The $\mathrm{p} 53$ protein and its signal transduction pathway respond to a wide variety of intrinsic and extrinsic cellular stresses. Among these stresses are DNA damage, telomere length shortening, hypoxia, reduction in ribonucleoside triphosphate pools and ribosomal biogenesis, spindle damage, and even mutational activation of oncogenes (Vogelstein et al. 2000). These stress signals are communicated to the p53 protein and its ubiquitin ligase, MDM2, via an extensive set of protein modifications that result in reduced expression or activity of the MDM2 protein, a negative regulator of p53 (Appella and Anderson 2001). In response to stress, the stability of p53 is increased and it becomes an active transcription factor. This in turn results in the transcription of a set of genes whose products bring about cell cycle arrest, senescence, or apoptosis. This provides time to either reverse the stress before cell duplication or kill the cell and its offspring that have duplicated under stressful conditions and made errors in that process. The replication of damaged DNA can increase the mutation rate by hundreds of fold. In this fashion, p53 and the signal transduction pathway it controls prevent errors in cell division that could give rise to mutations and changes in ploidy in a clone of cells. Several lines of evidence support this explanation; individuals that inherit a mutation in one of their $p 53$ alleles develop multiple cancers at a young age and at a high rate (Li 1990; Malkin et al. 1990), a set of phenotypes now known as Li-Fraumeni syndrome, and up to $50 \%$ of all cancers harbor somatic $p 53$ mutations that inactivate its function as a transcription factor (Soussi and Beroud 2001). Thus, there is a good deal of evidence that the p53 protein and its signal transduction pathway act in humans to suppress the development of tumors.

Several years ago, the sequencing of the human genome was completed, and it became clear that humans

${ }^{\dagger}$ These authors contributed equally to this work. are a young species, with any two individuals being 99.8\% identical (Lander et al. 2001; Venter et al. 2001). Among the differences between individuals are up to 10 million single-nucleotide polymorphisms (SNPs) distributed throughout the genome (Sachidanandam et al. 2001), and these differences may contribute to many of the individual traits that make us unique. This includes predispositions to diseases, responses to drugs or therapies, or even interactions with known mutations that predispose patients to diseases. Thus, we might anticipate that $p 53$, and those genes which encode the proteins that make up the p53 pathway, would contain SNPs which would either enhance or decrease the ability of this network to suppress tumor development. Indeed, some of these SNPs have been identified. There are two common alleles of $p 53$ that encode different amino acids at codon 72 , either proline or arginine, and allele frequencies within populations differ as one goes from the equator to northern latitudes (Beckman et al. 1994), suggesting selective forces play a role in maintaining both alleles. Indeed, the arginine form of p53 is associated with a stronger apoptotic response (Dumont et al. 2003), and the arginine-encoding allele of $p 53$ is preferentially mutated and maintained in squamous cell tumors (Marin et al. 2000). Recently, a SNP in the human MDM2 gene was shown to influence the number of independent cancers developed by Li-Fraumeni patients and to lower the age of onset of both familial and sporadic soft-tissue sarcomas (Bond et al. 2004).

To identify SNPs populating the p53 pathway that affect cancers in humans, we have used the candidate signal transduction pathway approach. From the growing list of genes within the p53 pathway, we have selected 82 genes and have identified 1335 SNPs within these genes. Although it might be possible to choose from among these SNPs those with the highest probability of altering the efficiency or functions of the p53 pathway, it became clear that a cell-based assay to test for the efficiency of 
p53 function should be developed which would permit an assessment of the impact of a SNP or gene upon the functions of the p53 pathway. This paper describes the development of an assay that, in addition to providing a method to demonstrate which SNPs alter the efficiency of the p53 pathway, provides cell lines that permit one to determine the molecular and cellular mechanism of the SNP. As the genotypes from multiple SNPs are determined within the same group of cell lines, this provides, for the first time, the chance to examine epistasis in the pathway and the combinatorial impact of several SNPs in the same cell. This assay has uncovered both sexual and racial dimorphisms in the p53 pathway.

The assay employed 113 EBV-transformed B-cell lines (LCLs) that were derived from unrelated, healthy volunteers. The input stress signal employed to activate the p53 protein was 5 Gy of gamma radiation $(\gamma$ IR), and the output or phenotype measured was the percentage of cells that underwent apoptosis 24 hours after the radiation. The results demonstrate that the p53 pathway plays a central role in determining this phenotype. Interestingly, each cell line undergoes a reproducible frequency of apoptosis with a small standard error, whereas the population responds with a range of $12.3-58.9 \%$ apoptosis around a median of $35.5 \%$ apoptosis. The tails of this distribution (high and low ends) are two standard deviations from the mean, providing a statistically robust method to characterize the distributions of any SNP in this population of cell lines. This assay has uncovered SNPs or haplotypes in two genes in the p53 pathway (Jin and Levine 2001): AKT1 and Perp, each of which alters the distribution of apoptotic response to irradiation. Because the cell lines are available to any investigator and have been previously classified for a phenotype (apoptosis after $\gamma$ IR), other researchers can now test for the impact of a SNP (or haplotype) by genotyping the DNA samples obtained from this collection. Furthermore, this approach can be adapted to many other phenotypes that can be measured in B cells in culture.

\section{MATERIALS AND METHODS}

Response of $E B V$-transformed $B$ cells to $\gamma$ radiation. A total of $120 \mathrm{EBV}$-transformed lymphoblast cell lines (LCLs) derived from anonymous, unrelated healthy volunteers were purchased from the Coriell Institute for Medical Research (Camden, New Jersey). Cells were cultured in RPMI supplemented with L-glutamine and 15\% FBS (Sigma). Cells were seeded into T25 flasks at a density of $2 \times 10^{5}$ cells $/ \mathrm{ml}$ and were grown at $37^{\circ} \mathrm{C}$ at $5 \%$ $\mathrm{CO}_{2}$ until the density reached $1 \times 10^{6}$ cells $/ \mathrm{ml}$ (defined as one passage). Once the cultures reached a viability of $90 \%$ or greater, response to radiation was measured. For each cell line, two $10-\mathrm{ml}$ cultures were seeded into T25 flasks at a density of $2 \times 10^{5}$ cells $/ \mathrm{ml}$ and were grown for 48 hours. One culture was irradiated with 5 Gy radiation (CIS BioInternational IBL $437 \mathrm{C}^{137} \mathrm{Cs} \quad \gamma$-radiation source), and the control culture was mock-irradiated. Cell death was measured in both cultures 24 hours post-radiation using a Guava Personal Cell Analysis flow cytome- ter (Hayward, California). Guava Viacount reagent, which differentially stains viable and nonviable cells based on their permeability to two dyes, was used to measure apoptosis. Control experiments on four LCLs demonstrated that assays of multicaspase activation, TUNEL staining and nexin staining, gave very similar results to Viacount (data not shown). The radiation-induced percent apoptotic cell death for a culture was obtained by comparison of the irradiated to the mock-treated control culture. The average apoptotic response for each cell line was calculated from at least three measurements, each taken from a different passage. The total number of cell lines in our analysis was reduced to 113 after elimination of data from cell lines that never reached $90 \%$ viability, grew very slowly, underwent crisis during the experiment, or did not give reproducible results in the assay.

Genotyping. Genomic DNAs corresponding to the 113 LCLs were purchased from Coriell Institute (Camden, New Jersey). The five polymorphisms in AKT1 (Emamian et al. 2004) (rs3803300, rs1130214, rs3730358, rs2498799, rs2494732) and the Perp polymorphism (rs2484067) were genotyped on an ABI Prism 7000 using Applied Biosystems (Foster City, California) allelic discrimination assays. All genotype frequencies were in Hardy-Weinberg equilibrium.

siRNA gene silencing. To prepare cells for transfection, LCLs were grown to a density of $1 \times 10^{6}$ cells $/ \mathrm{ml}$ and then centrifuged at $300 \mathrm{~g}$ for 10 minutes, and the medium was removed. Cells were resuspended at room temperature in Nucleofector solution included with the Nucleofector kit V (Amaxa, Maryland). A culture $(100 \mu \mathrm{l})$ at a density of $4 \times 10^{6}$ to $5 \times 10^{6}$ cells $/ \mathrm{ml}$ was mixed with siRNA and transferred to the provided cuvette and electroporated with an Amaxa Nucleofector device (Amaxa, Germany) set with the A-30 pulsing parameter, and cells were immediately transferred into a flask containing prewarmed $\left(37^{\circ} \mathrm{C}\right)$ culture medium. Transfection efficiency was determined by fluorescent microscopy using Alexa Fluor 546 control nonsilencing duplex siRNAs (Qiagen). Typical transfection efficiency was $70-80 \%$, and viability at 48 hours post-transfection was near $90 \%$. AKT1 siRNA Smartpool (Dharmacon) was used to silence $A k t 1$ expression. A p53 siRNA Smartpool (Dharmacon) was used to reduce $p 53$ expression. Lamin A/C and control nonsilencing duplex siRNAs (Qiagen) were used at the same concentration as $A K T 1$ and $p 53$ siRNAs (600 picomoles). For radiation studies of siRNA-transfected cells, cells were irradiated (5 Gy) 24 hours post-transfection, and percent cell death was measured 24 hours after radiation by comparing irradiated and mock-irradiated transfected cultures. Subsequently, the cells were lysed, and protein levels were analyzed as described below.

Western analysis. Protein levels were analyzed from total cell extracts prepared by lysis in RIPA buffer (Sigma Aldrich) in the presence of a protease and phosphatase inhibitor cocktail (Sigma Aldrich). Protein concentration was measured using Bio-Rad's protein assay and spec- 
trometry at $595 \mathrm{~nm}$. Total protein $(40 \mu \mathrm{g})$ was separated on a 4-20\% Tris-glycine gel and transferred to a PVDF membrane. The AKT1 (2H10), Lamin A/C (\#2032), and phospho-MDM2 Ser166 (\#3521) antibodies were purchased from Cell Signaling Tech, $\alpha$-Tubulin (DM1A) antibody was from Sigma Aldrich, the p53 (DO-1) and MDM2 (SMP14) antibodies were from Santa Cruz.

The proteins were visualized using HRP-conjugated secondary antibodies (Pierce Biotech) and SuperSignal West Fento (Pierce Biotech).

Determination of AKT1 protein concentration by $\boldsymbol{E L I S A}$. For each cell line, cultures were seeded into T25 flasks at a density of $2 \times 10^{5}$ cells $/ \mathrm{ml}$ and were grown for 72 hours. Total cell protein extracts were prepared and quantitated as described above. AKT1 protein in each extract was determined using the PathScan Total AKT1 Sandwich ELISA kit from Cell Signaling Technology (Beverly, Massachusetts) according to the manufacturer's recommendations. The results of the ELISA assay were confirmed by western analysis (data not shown).

$\boldsymbol{R N A}$ analysis. To measure basal Perp mRNA levels, total RNA was isolated from cell pellets using Rneasy (Qiagen). cDNAs were made using TaqMan reverse transcription reagents from Applied Biosystems. Real-time PCR was carried out on an ABI Prism 7000 sequence detection system. Probe and primer sets were as follows: human Perp (catalog number Hs00751717_s1) and human GAPDH (catalog number Hs99999905_m1) (Applied Biosystems).

Statistical analysis. Means and standard deviations of total protein were compared by unpaired two-tailed $t$ tests. Means and standard deviations of apoptotic responses of cell lines were compared by unpaired $t$-tests, one- or two-tailed as indicated in the text, or by the Fisher's exact test when appropriate.

Determination of haplotype structure. Haplotypes were determined by Expectation Maximization using the SNPHAP software (www-gene.cimr.cam.as.uk/clayton).

\section{RESULTS \\ The Response of LCLs to Radiation Is Heterogeneous}

To measure the response of cells from different individuals to DNA damage, we chose to irradiate EBVtransformed B cells derived from those individuals. It has been demonstrated in both mice (Lowe et al. 1993) and humans (Camplejohn et al. 2000) that apoptotic responses in lymphocytes are significantly reduced by $p 53$ mutations. EBV-transformed B-cell lines were chosen to reduce the cell type heterogeneity and environmental variables that are observed when peripheral blood lymphocytes are taken directly from an individual and placed in culture (Camplejohn et al. 2003). In addition, the LCLs provide a permanent source of cells that can be tested multiple times for a given phenotype or phenotypes. Of the 113 LCLs included in the study, 77 are derived from females and 36 from males. To increase the genetic diversity, roughly half (58) of the LCLs are derived from African-American donors and half (55) are from Caucasian donors. Each cell line was irradiated with 5 Gy of gamma radiation $(\gamma$ IR), and 24 hours later the percent of cells in the culture undergoing apoptotic cell death was measured. Radiation-induced apoptosis was measured at least three times for each LCL. The average standard error was $2.2 \%$ apoptosis and ranged from $0.2 \%$ to $5.3 \%$. Thus, the response of each individual LCL was very reproducible. When the percent of apoptosis was measured at 48 hours, the frequency of apoptosis increased, but the relative order of responses of different LCLs remained substantially the same. In Figure 1, data for the $113 \mathrm{LCLs}$ are plotted as the number of cell lines as a function of the frequency of apoptosis at 24 hours. Response varied with a range of $12.3-58.9 \%$ apoptosis forming a Gaussian distribution with an average apoptotic frequency of $34.9 \%$, a median of $35.5 \%$, and a standard deviation of $9.9 \%$. On the basis of the standard error and the standard deviation, we chose to bin the responses into $10 \%$ intervals of apoptosis (Fig. 1). The midpoints of the highest (8 LCLs) and lowest (11 LCLs) bins of this distribution are two standard deviations removed from the median. This experiment demonstrates that heterogeneity exists in the response of individual B-cell lines to DNA damage induced by $\gamma$ IR. In addition, these data suggest that it should be possible to identify, in a statistically valid fashion, individual traits or SNPs that generate some of this variation.

Although the presence of Epstein-Barr virus in these cells may alter some of their properties, it is unlikely that the distribution of apoptotic response is drastically altered. The results presented in Figure 1 are similar to those of Camplejohn (Camplejohn et al. 2003) and K. Onel and A.J. Levine (unpubl.), whereby peripheral blood lymphocytes, isolated directly from donors, were tested in a similar assay. Second, when an EBV-transformed LCL derived from a Li-Fraumeni patient (cell line 051-019) was tested in this assay, the frequency of apoptosis was only $13.3 \%$ (Fig. 2A), similar to that found using peripheral blood lymphocytes from Li-Fraumeni pa-

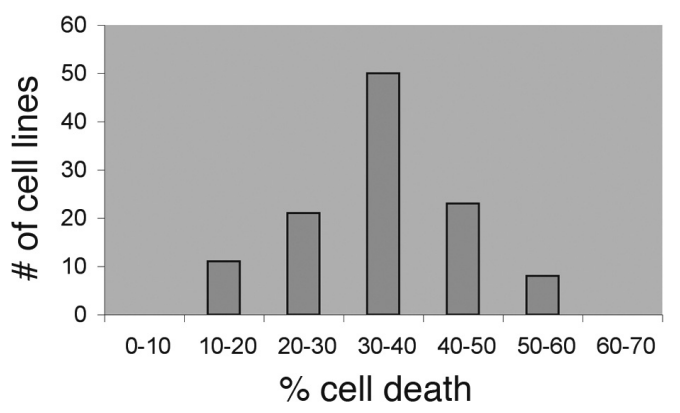

Figure 1. The response of LCLs to radiation is heterogeneous. 113 LCLs from unrelated individuals were irradiated with 5 Gy $\gamma$ IR to induce DNA damage, and apoptosis was measured $24 \mathrm{hr}$ later. Each cell line was measured at least three times; the average standard error for all cell lines was $2.2 \%$. Each cell line was placed in a bin based on its average apoptotic response. The bin size of $10 \%$ represents one standard deviation $(9.9 \%)$ for the population. 
A

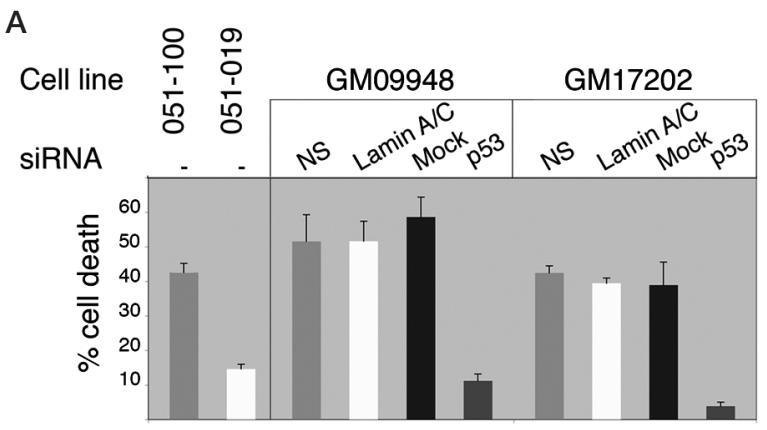

B
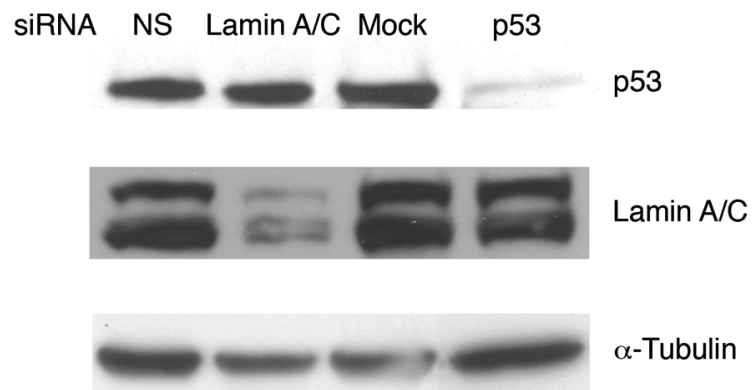

Figure 2. Reduction in p53 function results in reduced apoptotic response in LCLs. (A) An LCL (051-019) derived from a patient with Li-Fraumeni syndrome ( $p 53$ mutant) and an LCL from an unrelated individual with wild-type $p 53$ alleles (051-100) were irradiated with 5 Gy $\gamma$ IR to induce DNA damage; apoptotic cell death was measured $24 \mathrm{hr}$ later. Each cell line was measured four times; the average cell death and standard error are shown. LCLs GM09948 and GM17202 were transfected with siRNAs specific for $p 53$, lamin $A / C$, or a nonspecific siRNA (NS). Cells were treated with $\gamma$ IR $24 \mathrm{hr}$ after transfection with siRNAs, and cell death was measured $24 \mathrm{hr}$ post-radiation. Shown are the mean and standard deviation from two separate experiments. $(B)$ Shown are western blots of basal (no radiation) whole-cell extracts made from the GM09948 cell line $48 \mathrm{hr}$ post-transfection with the indicated siRNAs.

tients (Camplejohn and Rutherford 2001) and markedly different from a second LCL (051-100) derived from a volunteer with wild-type $p 53$ that underwent considerable apoptosis (44\%). To further demonstrate the central role of the p53 pathway in this assay, two LCLs with high apoptotic responses were transfected with $p 53$ siRNA to reduce the expression of $p 53$ and test whether this would affect the efficiency of apoptosis. As shown in Figure 2A, transfection of the GM09948 cell line, shown previously to have an apoptotic frequency of $53.6 \%$, with a nonspecific siRNA or with a siRNA directed against lamin, resulted in no change in the response to $\gamma$ IR. Transfection of the same cell line with a $p 53$ siRNA that lowered the amount of $\mathrm{p} 53$ expression (Fig. 2B) resulted in a reduction of apoptotic response to about 10\% (Fig. 2A). A similar result was obtained with a second LCL, GM17202; $p 53$ siRNA reduced apoptosis from $44.4 \%$ to below $10 \%$ (Fig. 2A). It is clear from these experiments that this assay measures p53-mediated apoptosis in response to $\gamma$ IR and that the efficiency of that response is quite heterogeneous in the population.

\section{The Genetic Background of the LCLs Plays a Role in the Heterogeneity of Their Responses to DNA Damage}

Of the 113 cell lines shown in Figure 1, 55 are derived from the B cells of self-identified Caucasians and 58 are derived from self-identified African-American donors. The LCLs were chosen this way to maximize the number of polymorphisms that would be present. The African and African-American populations have been shown to have a greater number of unique or population-specific polymorphisms compared to the European Caucasian population (Hinds et al. 2005). Figure 3 presents the 113 cell lines separated by the race of the donor as a function of apoptotic cell death. Both the Caucasian and the AfricanAmerican LCLs produced a similar normal distribution of responses between $20 \%$ and $60 \%$ apoptosis, but all of the 11 LCLs exhibiting between $10 \%$ and $20 \%$ apoptosis were of Caucasian origin ( $p=0.0002$; Fisher's exact test, two-tailed). This significant overrepresentation of Caucasians in the lowest response group is seen for both female and male LCLs $(p=0.005$ and 0.04 , Fisher's exact, two-tailed, respectively). Since the culture conditions employed in this assay are relatively constant for all cell lines, these data support the idea that genetic factors are contributing to the heterogeneity of the $\mathrm{p} 53$ response to radiation damage.

\section{A Haplotype in $A K T 1$ Affects Radiation-induced Cell Death}

Recently, a haplotype composed of five SNPs in AKT1 was reported to reduce the amount of AKT1 protein in lymphocytes and brain tissue of schizophrenic patients, and this haplotype was found in higher than expected frequencies in individuals with schizophrenia (Emamian et al. 2004). AKT1 is an antiapoptotic protein kinase (Bellacosa et al. 2004), and one of its substrates is the MDM2 protein (Mayo and Donner 2001; Zhou et al. 2001; Gottlieb et al. 2002; Ogawara et al. 2002). Phosphorylation of MDM2 by AKT1 at serine residues 166 and 188 inhibits MDM2 autoubiquitination leading to the stabilization of MDM2 (Ogawara et al. 2002; Feng et al. 2004). In some cells, phosphorylation also promotes the movement of MDM2 into the nucleus (Zhou et al. 2001) where it can act to destabilize p53 protein levels and lower apoptosis. Thus, AKT1

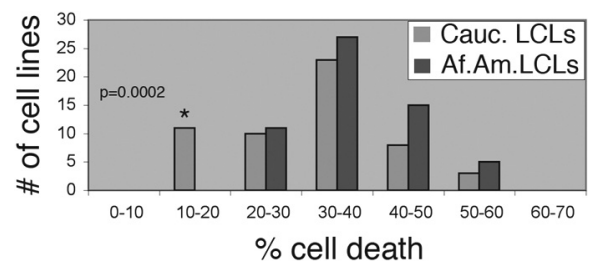

Figure 3. The response of LCLs to radiation is influenced by genetic factors. LCLs are grouped by average response to radiation and by the race of the donor. 55 LCLs are derived from selfidentified Caucasian donors, and 58 are derived from self-identified African-American donors. 
can act epistatically to MDM2 and functions to control the amount of MDM2 in a cell. To determine whether AKT1 activity affects apoptotic frequency in B cells, two LCLs (both in the lowest response group) were transfected with siRNAs that were either nonspecific, directed against Lamin, or directed against the AKT1 transcript. Transfection with $A K T 1$ siRNA lowered the levels of AKT1 protein, lamin siRNA reduced the expression of the lamin protein, and so both of these siRNAs were specific for their targets (Fig. 4B). Knock-down of AKT1 resulted in an increased apoptotic response (37\% and $38 \%$ ) compared to mock or control-treated transfectants $(11 \%$ and $12 \%$ apoptosis) (Fig. 4A). These data suggest that $A K T 1$ expression can affect the frequency of apoptosis in B cells.

The LCLs were genotyped for five SNPs in the AKT1 gene (Emamian et al. 2004), and their positions are diagramed in Figure 5A. The pair-wise linkage disequilibrium (LD) is presented as $\mathrm{D}^{\prime}$. A D' value of 1.0 indicates complete LD, whereas a $\mathrm{D}^{\prime}$ value of 0 indicates that historical recombination events have occurred between the two SNPs such that they are only randomly present on the same chromosome. The predicted haplotypes and their calculated frequencies are presented in Table 1. AKT1 SNP3 and SNP4 are in almost complete LD in Caucasians but are not in LD in the African-American population. Because haplotypes that include SNP3 have been

A

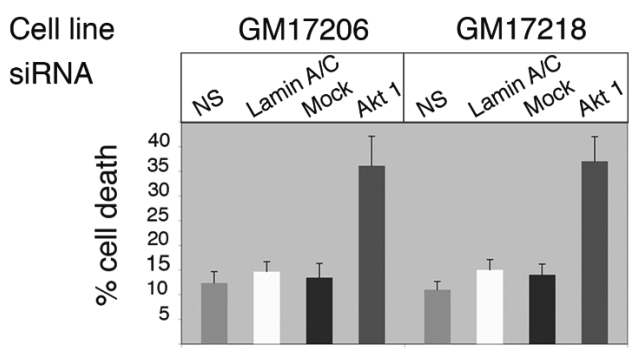

B

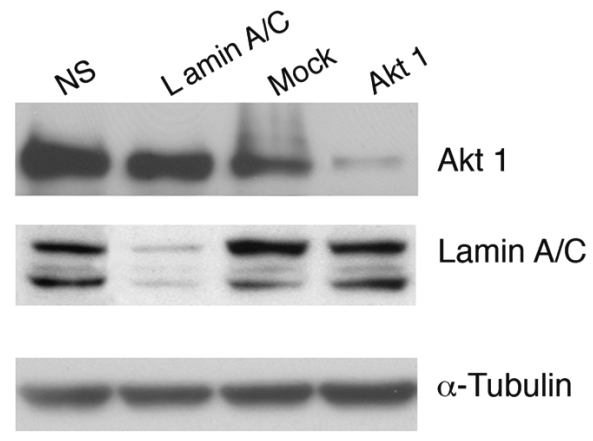

Figure 4. Reduction in expression of $A K T 1$ by siRNA results in increased apoptotic response in LCLs. (A) LCLs GM17206 and GM17218 were transfected with siRNAs specific for $A K T 1$, lamin $A / C$, or a nonspecific siRNA (NS). Cells were treated with $\gamma$ IR (5 Gy) $24 \mathrm{hr}$ after transfection with siRNAs, and apoptotic cell death was measured $24 \mathrm{hr}$ post-radiation. Shown are the mean and standard deviation from three separate experiments. $(B)$ Shown are western blots of basal (no radiation) whole-cell extracts made from cell line GM17206 $48 \mathrm{hr}$ post-transfection with the indicated siRNAs. shown to associate with the amount of AKT1 protein in lymphocytes, and our data show that these SNPs are linked in Caucasians but not in African-Americans, we can make the prediction that a core SNP3-SNP4 haplotype in $A K T 1$ will be associated with apoptosis in Caucasian LCLs but not in African-American LCLs and that this haplotype may have a significant impact on the response of the 11 Caucasian cell lines in the lowest responding group.

In Figure 5B, 50 Caucasian LCLs are separated on the basis of their genotypes at AKT1 SNP3 and SNP4 and their response to $\gamma$ IR. LCLs homozygous for the minor alleles at both SNP3 (TT) and SNP4 (AA) populate the high response end of the distribution, LCLs heterozygous for both SNP3 (CT) and SNP4 (GA) populate the middle of the distribution, whereas those LCLs homozygous for the major alleles at both SNP3 (CC) and SNP4 (GG) are found in a broad or biphasic distribution encompassing the lower half of the graph that includes the 11 lowest apoptotic LCLs. The Caucasian LCLs homozygous for the major alleles (SNP3 CC, SNP4 GG) undergo, on average, less apoptosis in response to $\gamma$ IR than do the heterozygous (CT, GA) LCLs ( $p=0.017, t$-test, one-tailed). A similar comparison of the African-American homozygous (CC, GG) LCLs to the heterozygous (CT, GA) LCLs reveals that these genotypes are not associated with apoptosis in this group ( $p=0.95, t$-test, two-tailed). All 11 of the Caucasian LCLs in the 10-20\% apoptotic response group share the major SNP3-SNP4 haplotype (CC, GG) ( $p=0.014$, Fisher's exact, one-tailed), suggesting that this haplotype is necessary, but not sufficient to lower the apoptotic index of LCLs to the 10-20\% range because the same haplotype populates LCLs in the $20-30 \%$ and $30-40 \%$ region of the distribution in Figure 5B. Notably, the broad distribution of cell death for Caucasian LCLs with the major AKT1 SNP3-SNP4 haplotype suggests that the low-apoptosis phenotype may be modified by other genetic factors.

Because AKT1 is an antiapoptotic kinase, we predicted that the LCLs with the highest apoptotic responses would exhibit the lowest levels of cellular AKT1 protein and, conversely, LCLs with the lowest responses to $\gamma$ IR would have the highest levels of AKT1 protein or activity. As shown in Figure 5C, this is indeed true. Caucasian LCLs that are homozygous for the major SNP3-SNP4 haplotype $(\mathrm{CC} / \mathrm{GG})$ have higher amounts of AKT1 protein than do the LCLs harboring the minor haplotype (TT/AA) ( $p$ $=8.0 \times 10^{-8}$, unpaired $t$-test, two-tailed), and LCLs that are heterozygous at both positions have intermediate amounts of AKT1 protein $\left(p=1.7 \times 10^{-9}\right)$. Thus, it appears that the SNP3-SNP4 haplotype controls the level of AKT1 in lymphocytes and affects the magnitude of a cell's response to DNA damage.

To determine whether the level of AKT1 affects apoptosis by altering MDM2 stability, AKT1 expression was reduced in an LCL by siRNA. LCL GM17206 was transfected with siRNAs for AKT1, Lamin, and a nonspecific siRNA. Cultures were split after transfection, and half of each culture was irradiated (5 Gy) and half remained untreated. 24 hours post-radiation, the cells were harvested 
A

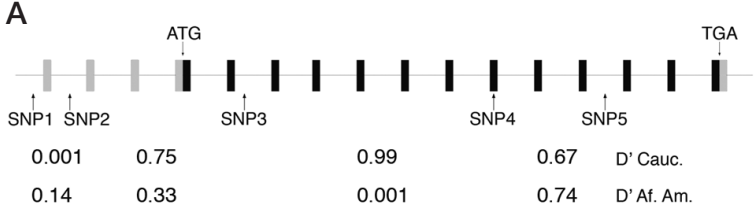

B

Figure 5. The major $A K T 1$ SNP3-4 haplotype is associated with higher basal AKT1 protein levels and decreased apoptotic response. (A) Genomic structure of the AKT1 locus and polymorphic sites used. Exons (boxes) and introns are not drawn to scale; gray boxes represent noncoding sequences, black boxes represent coding sequences. Pair-wise linkage disequilibrium between the SNPs calculated as D' is shown. Allele frequencies for individual SNPs are as follows: (Caucasian) SNP1 $(\mathrm{A}=94.5, \mathrm{G}=5.5) \mathrm{SNP} 2$ $(\mathrm{G}=70.0, \mathrm{~T}=30.0) \mathrm{SNP} 3(\mathrm{C}=88.2, \mathrm{~T}=11.8) \mathrm{SNP} 4(\mathrm{G}=81.8$, $\mathrm{A}=18.2) \mathrm{SNP} 5(\mathrm{~A}=60.9, \mathrm{G}=39.1)$; African-American SNP1 (A $=75.0, \mathrm{G}=25.0) \mathrm{SNP} 2(\mathrm{G}=64.7, \mathrm{~T}=35.3) \mathrm{SNP} 3(\mathrm{C}=83.6, \mathrm{~T}$ $=16.4) \mathrm{SNP} 4(\mathrm{G}=85.3, \mathrm{~A}=14.7) \mathrm{SNP} 5(\mathrm{~A}=45.7, \mathrm{G}=54.3)$. (B) The major $A K T 1$ haplotype $(\mathrm{CC} / \mathrm{GG})$ is associated with lower apoptosis. 50 Caucasian LCLs are grouped by average response to radiation and genotype at $A K T 1$ SNPs 3 and 4. Five cell lines were eliminated from the analysis because they had AKT1 SNP34 genotype combinations other than those shown in Fig. 5B. (C) The AKT1 major SNP3-4 haplotype is associated with higher basal AKT1 protein levels. Mean \pm S.E.M. optical densities from an AKT1-specific ELISA are shown. The data include the results from two separate experiments. $(D)$ Reduction in expression of $A K T 1$ by siRNA results in decreased phosphorylation and stability of MDM2. GM17206 was transfected with siRNAs specific for $A K T 1$, lamin $A / C$, or a nonspecific siRNA (NS). Transfected cells were exposed to $\gamma$ IR (5 Gy) 24 hours after transfection. Shown is a western blot of whole-cell protein extracts that were prepared $24 \mathrm{hr}$ after irradiation.

and prepared for western blot analysis employing antibodies that detected total MDM2 protein, MDM2 phospho-Ser-166, and total $\mathrm{p} 53$. These results are presented in Figure 5D. The $A K T 1$ - and lamin-specific siRNAs acted in a gene-specific fashion to reduce expression of each gene product accordingly. As expected, because activation of $\mathrm{p} 53$ by DNA-damage-induced signals leads to increased expression of the $\mathrm{p} 53$-target gene $M D M 2$, radiation increased the concentration of MDM2 in the mock-treated, lamin siRNA transfected, and the nonspecific siRNA transfected cells. In irradiated cells with reduced $A K T 1$ expression, MDM2 levels were much lower, and phosphorylation of MDM2 at Ser-166 was much reduced. At the same time, p53 levels were elevated in the $A K T 1$ siRNA transfected cells. This supports the hypoth-

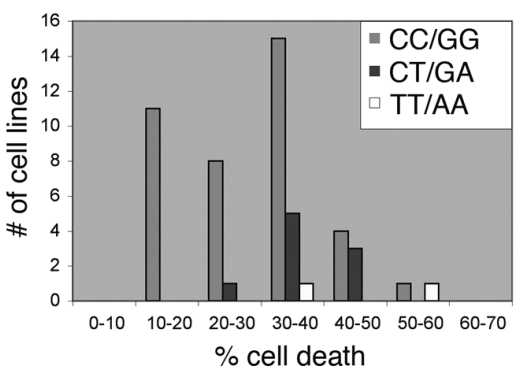

C

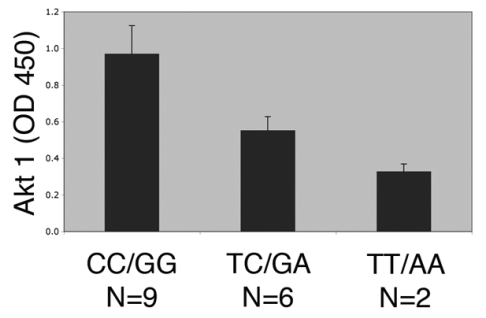

D

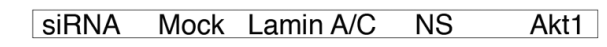

Gamma R - + - + - $\quad+\quad-\quad+$

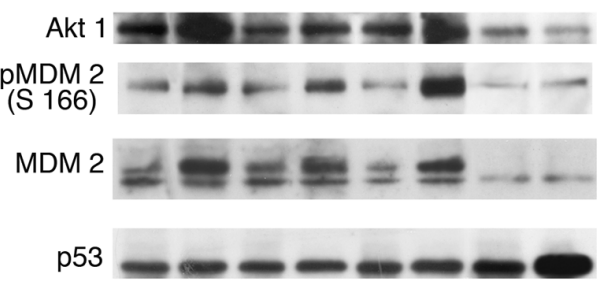

Lamin A/C

$\alpha$-Tubulin

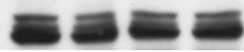

esis that AKT1 functions in B cells to phosphorylate and stabilize MDM2, leading to the decreased stability and activity of $\mathrm{p} 53$.

Table 1. Haplotype Frequencies for $A K T 1$ SNP1-5 in Caucasians and African-Americans

\begin{tabular}{ccccc}
\hline \multicolumn{2}{c}{ Caucasian } & & \multicolumn{2}{c}{ African-American } \\
\cline { 1 - 2 } \cline { 5 - 5 } haplotype & frequency & & haplotype & frequency \\
\hline AGCGA & 0.53 & & AGCGG & 0.21 \\
ATCGG & 0.14 & & AGCGA & 0.19 \\
ATTAG & 0.098 & & GTCGG & 0.088 \\
AGCGG & 0.054 & & ATCGA & 0.084 \\
GGCGG & 0.039 & & GGCGG & 0.075 \\
AGCAA & 0.037 & & ATTGA & 0.074 \\
ATCGA & 0.033 & & AGCAG & 0.070 \\
& & & GGTGA & 0.032 \\
\hline
\end{tabular}


In African-Americans, SNP3 and 4 are not in linkage disequilibrium, and thus it is not possible to define a SNP3-SNP4 haplotype in this population. However, examined independently, SNP3 ( $p=0.63, t$-test, two-tailed) and SNP4 ( $p=0.90, t$-test, two-tailed) appear to make no contribution to the apoptotic response of African-American LCLs. Thus, it appears that the functional SNP in $A K T 1$ (as yet unidentified) that results in altered apoptosis is not in LD with either SNP3 or SNP4 in the AfricanAmerican population, whereas it is in LD with these SNPs in the Caucasian population. Alternatively, the same functional SNP may be present in both groups but may occur at a much lower frequency in the AfricanAmerican population.

\section{A SNP in Perp Affects Response to Radiation in LCLs}

Having established that measurement of apoptotic frequency in LCLs provides a good assay for identifying SNPs in genes that both lower the apoptotic index of a cell line and have clinical correlates that reflect this phenotype, this approach was employed to test a number of SNPs in genes that populate the p53 pathway, and this led to the identification of a SNP in Perp. Perp is a p53-inducible gene whose transcript is increased in p53-activated cells undergoing apoptosis, but not in cells undergoing p53-mediated $\mathrm{G}_{1}$ growth arrest (Attardi et al. 2000). Perp is a tetraspanin membrane protein whose functions include the promotion of apoptosis in lymphocytes and neuronal cells, adhesion of epithelial cells, and the development of skin and notochord (Ihrie and Attardi 2004; Ihrie et al. 2005; Nowak et al. 2005). The overexpression of the Perp protein in cells will induce apoptosis even in the absence of a p53 response (Ihrie et al. 2003). The SNP in Perp that we have identified is located in the second intron, 76 nucleotides 3 ' to the boundary of exon 2 , and the SNP changes a $G$ residue to an A residue (IVS2 $+76 \mathrm{G}>\mathrm{A}$ ). This polymorphism will be referred to as Perp SNP 2-76. This SNP may be in linkage disequilibrium with several other SNPs, forming a haplotype that gives rise to the mechanism of action to be described here. The genotypes for this SNP occur at different frequencies in Caucasians (AA, 20\%; AG, 57\%; GG, 23\%) and AfricanAmericans (AA, 56\%; AG, 37\%; GG, 7\%). In female LCLs, homozygosity for the G allele of Perp SNP 2-76 is closely correlated with a low apoptotic frequency $(p=$ 0.0000021 ; $t$-test, two-tailed) as shown in Figure 6A. The $\mathrm{G}$ allele appears to act in a recessive fashion because LCLs with the AG genotype behave the same as LCLs with the AA genotype. Independently, Caucasian female LCLs and African-American female LCLs each show a significant correlation of the GG genotype with low frequencies of apoptosis ( $p=0.002$ and 0.004 , respectively, $t$-test, two tailed). Thus, the polymorphism acts independently of race. In contrast, this SNP has no significant impact on apoptosis in male LCLs $(p=0.39)$, and so Perp SNP 2-76, like the MDM2 SNP309, shows a sexual dimorphism whereby females are affected and not males. The Perp SNP 2-76 GG genotype appears to contribute to the phenotype of the seven female LCLs that populate the low-response group (10-20\% apoptosis) observed in Figures 1 and 3. Six of the seven female LCLs in this bin are GG at Perp SNP 2-76 ( $p=0.001$, Fisher's exact test, twotailed).

The basal levels of Perp transcript were determined, using real-time PCR, in 17 female cell lines that were AA (6), AG (5), or GG (6), and the results of this analysis are presented in Figure 6B. Interestingly, the basal amounts of Perp mRNA from AA and AG cell lines were similar, whereas the GG cell lines had about 2-3-fold lower expression of Perp. A comparison of the Perp transcript expressed in the 11 measured LCLs that have one or two copies of the A allele to the 6 LCLs with the GG genotype reveals that this difference is significant $(p=0.01, t$-test, one-tailed). Again, this suggests that the $G$ allele is recessive to the A allele. Thus, low expression of Perp and a reduced apoptotic response correlate well with the Perp SNP 2-76 GG genotype. The mechanism that may contribute to this result remains to be elucidated.

\section{DISCUSSION}

One of the immediate benefits from the DNA sequence and map of the human genome was the identification of SNPs that can affect or modify a wide variety of diseases. Today there are almost ten million human SNPs annotated in several databases, and a number of approaches have been devised to identify which SNPs might have a

A

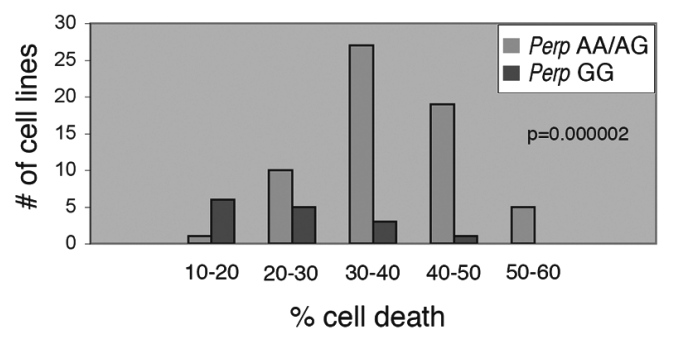

B

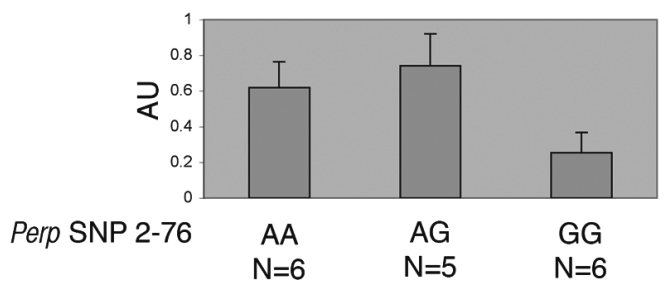

Figure 6. Female LCLs homozygous for a polymorphism in Perp, SNP 2-76 exhibit reduced response to DNA damage. $(A)$ Female LCLs grouped by average response to radiation and genotype at Perp SNP 2-76. LCLs homozygous for the G allele at Perp SNP 2-76 (GG) are grouped together; heterozygous (AG) LCLs are grouped with LCLs homozygous for the A allele at Perp SNP 2-76 (AA). (B) The average basal levels of Perp RNA from LCLs with AA, AG, or GG genotypes at Perp SNP 2-76 are shown as measured by real-time PCR (Taqman). All values (arbitrary units) have been normalized to the level of GAPDH and are the average of three independent measurements. The standard errors are shown. 
phenotype that could correlate with a clinical outcome. We have chosen to narrow the number of SNPs for study by developing several guidelines for our choice of SNPs: (1) Identify SNPs in genes that populate a signal transduction pathway which is clearly involved in a disease process (the candidate pathway approach). (2) Where possible, select genes at central nodes in the pathway that have the greatest capacity to affect the functions of the pathway. (3) Select SNPs that occur at a reasonably high frequency in a population to facilitate clinical testing. (4) Where possible, identify SNPs that may alter the expression or function of the gene products in the pathway. (5) Develop an assay that can test the functional significance of a SNP in cell culture. This paper describes an assay employing lymphoblastoid cell lines in culture that measures the efficiency of p53-mediated apoptosis in the population. The assay is reproducible and has been used to identify SNPs in p53 pathway genes that affect one of the functions (apoptosis) of this network that is central to the development of cancer. This assay has several advantages for future studies. Using the apoptotic frequencies in Figure 1, all one has to do to test for a functional SNP is to genotype the DNA from these cell lines. In addition, this assay can be adapted to any input (DNA damage, response to hormones, etc.) or the measurement of other outputs (phenotypes) which are reproducible and important to clinical outcomes. This assay has also elucidated several properties of the p53 pathway for which there were some indications in the literature. The ability to employ siRNA knock-down experiments in selected LCLs confirmed, at the molecular level, that AKT1 acts on MDM2 to increase its stability and thereby reduce p53mediated apoptosis in lymphocytes, thus demonstrating epistatic relationships between the AKT1 and MDM2 genes. AKT1 haplotype analysis has helped to explain at least a portion of the racial dimorphism observed in this assay. Linkage disequilibrium of $A K T 1$ SNP3 and SNP4 is only observed in LCLs derived from Caucasians, and all of the LCLs within the lowest apoptotic response group harbor the same major haplotype. Thus, the major AKT1 SNP3-SNP4 haplotype is necessary but not sufficient to produce the low response seen in this group. Additional SNPs remain to be uncovered. In addition to these demonstrated epistatic relationships and racial dimorphisms, sexual dimorphism in the behavior of the Perp SNP 2-76 was demonstrated. This SNP functions to reduce apoptosis in female LCLs to a much greater extent than in male LCLs. However, we analyzed many more LCLs derived from females $(n=77)$ than from males $(n$ $=36$ ), and so this conclusion will await some confirmation. However, sexual dimorphism has been observed in the $\mathrm{p} 53$ pathway previously. In $p 53$ mutant mice, the incidence of osteosarcoma was observed to be consistently higher in female mice than in male mice, regardless of genetic background (Donehower et al. 1995). In humans with Li-Fraumeni syndrome, tumors appear at higher frequencies in females than in males, even eliminating breast cancers from the calculation (Hwang et al. 2003). Both of these phenotypes could relate to SNPs that can lower the efficiency of apoptosis in females to a greater extent than in males.
Examination of the role of these SNPs provides a number of important lessons about the p53 pathway. Complexity will result from the epistatic relationships of genes and their products in a signal transduction pathway, as well as from the sexual dimorphism observed in the p53 pathway. Sexual dimorphism may arise from the presence of sex-hormone-specific transcription-factorbinding sites in the genes under study, and polymorphisms may create or destroy such sites. Sexual dimorphism can also arise from transcription-factor-binding sites that bind transcription factors encoded by genes located on the Y chromosome. It is notable that the SNPs presented here in AKT1 and Perp are located in potential regulatory regions of genes and result in altered expression of mRNA or protein. Second, it is important to point out that the phenotype of the p53 pathway that was explored in this study, in vitro apoptosis, may not be the only relevant phenotype when translating cell culture studies to in vivo tests. The p53 pathway is thought to act as a checkpoint for the surveillance of physical stress such as DNA damage. The loss of this checkpoint results in a high mutation rate and the increased likelihood of early-onset tumors and the development of multiple independent tumors. We have demonstrated that SNPs in the genes that populate the $\mathrm{p} 53$ pathway will combine to determine the efficiency of this surveillance system.

\section{ACKNOWLEDGMENTS}

This work was supported by grants to A. Levine from the Breast Cancer Research Foundation and the National Institutes of Health (5P01CA087497).

\section{REFERENCES}

Appella E. and Anderson C.W. 2001. Post-translational modifications and activation of $\mathrm{p} 53$ by genotoxic stresses. Eur. J. Biochem. 268: 2764.

Attardi L.D., Reczek E.E., Cosmas C., Demicco E.G., McCurrach M.E., Lowe S.W., and Jacks T. 2000. PERP, an apoptosis-associated target of p53, is a novel member of the PMP22/gas3 family. Genes Dev. 14: 704.

Beckman G., Birgander R., Sjalander A., Saha N, Holmberg P.A., Kivela A., and Beckman L. 1994. Is p53 polymorphism maintained by natural selection? Hum. Hered. 44: 266.

Bellacosa A., Testa J.R., Moore R., and Larue L. 2004. A portrait of AKT kinases: Human cancer and animal models depict a family with strong individualities. Cancer Biol. Ther. 3: 268 .

Camplejohn R.S. and Rutherford J. 2001. p53 functional assays: Detecting p53 mutations in both the germline and in sporadic tumours. Cell Prolif. 34: 1.

Camplejohn R.S., Sodha N., Gilchrist R., Lomax M.E., Duddy P.M., Miner C., Alarcon-Gonzalez P., Barnes D.M., and Eeles R.A. 2000. The value of rapid functional assays of germline p53 status in LFS and LFL families. Br. J. Cancer 82: 1145 .

Camplejohn R.S., Gilchrist R., Easton D., McKenzie-Edwards E., Barnes D.M., Eccles D.M., Ardern-Jones A., Hodgson S.V., Duddy P.M., and Eeles R.A. 2003. Apoptosis, ageing and cancer susceptibility. Br. J. Cancer 88: 487.

Donehower L.A., Harvey M., Vogel H., McArthur M.J., Montgomery C.A., Jr., Park S.H., Thompson T., Ford R.J., and Bradley A. 1995. Effects of genetic background on tumorigenesis in p53-deficient mice. Mol. Carcinog. 14: 16.

Dumont P., Leu J.I., Della Pietra A.C., III, George D.L., and Murphy M. 2003. The codon 72 polymorphic variants of $\mathrm{p} 53$ 
have markedly different apoptotic potential. Nat. Genet. 33: 357.

Emamian E.S., Hall D., Birnbaum M.J., Karayiorgou M., and Gogos J.A. 2004. Convergent evidence for impaired AKT1GSK3beta signaling in schizophrenia. Nat. Genet. 36: 131.

Feng J., Tamaskovic R., Yang Z., Brazil D.P., Merlo A., Hess D., and Hemmings B.A. 2004. Stabilization of Mdm2 via decreased ubiquitination is mediated by protein kinase B/Aktdependent phosphorylation. J. Biol. Chem. 279: 35510.

Gottlieb T.M., Leal J.F., Seger R., Taya Y., and Oren M. 2002. Cross-talk between Akt, p53 and Mdm2: Possible implications for the regulation of apoptosis. Oncogene 21: 1299.

Hinds D.A., Stuve L.L., Nilsen G.B., Halperin E., Eskin E., Ballinger D.G., Frazer K.A., and Cox D.R. 2005. Wholegenome patterns of common DNA variation in three human populations. Science 307: 1072.

Hwang S.J., Lozano G., Amos C.I., and Strong L.C. 2003. Germline p53 mutations in a cohort with childhood sarcoma: Sex differences in cancer risk. Am. J. Hum. Genet. 72: 975.

Ihrie R.A. and Attardi L.D. 2004. Perp-etrating p53-dependent apoptosis. Cell Cycle 3: 267.

Ihrie R.A., Reczek E., Horner J.S., Khachatrian L., Sage J., Jacks T., and Attardi L.D. 2003. Perp is a mediator of p53-dependent apoptosis in diverse cell types. Curr. Biol. 13: 1985.

Ihrie R.A., Marques M.R., Nguyen B.T., Horner J.S., Papazoglu C., Bronson R.T., Mills A.A., and Attardi L.D. 2005. Perp is a p63-regulated gene essential for epithelial integrity. Cell 120: 843 .

Jin S. and Levine A.J. 2001. The p53 functional circuit. J. Cell Sci. 114: 4139

Lander E.S., Linton L.M., Birren B., Nusbaum C., Zody M.C., Baldwin J., Devon K., Dewar K., Doyle M., FitzHugh W., Funke R., Gage D., Harris K., Heaford A., Howland J., Kann L., Lehoczky J., LeVine R., McEwan P., McKernan K., Meldrim J., Mesirov J.P., Miranda C., Morris W., and Naylor J., et al. (International Human Genome Sequencing Consortium). 2001. Initial sequencing and analysis of the human genome. Nature 409: 860 .

Li F.P. 1990. Familial cancer syndromes and clusters. Curr. Probl. Cancer 14: 73.

Lowe S.W., Schmitt E.M., Smith S.W., Osborne B.A., and Jacks T. 1993. p53 is required for radiation-induced apoptosis in mouse thymocytes. Nature 362: 847.

Malkin D., Li F.P., Strong L.C., Fraumeni J.F., Jr., Nelson C.E.,
Kim D.H., Kassel J., Gryka M.A., Bischoff F.Z., and Tainsky M.A., et al. 1990. Germ line p53 mutations in a familial syndrome of breast cancer, sarcomas, and other neoplasms. Science 250: 1233-8.

Marin M.C., Jost C.A., Brooks L.A., Irwin M.S., O’Nions J., Tidy J.A., James N., McGregor J.M., Harwood C.A., Yulug I.G., Vousden K.H., Allday M.J., Gusterson B., Ikawa S., Hinds P.W., Crook T., and Kaelin W.G., Jr. 2000. A common polymorphism acts as an intragenic modifier of mutant p53 behaviour. Nat. Genet. 25: 47.

Mayo L.D. and Donner D.B. 2001. A phosphatidylinositol 3-kinase/Akt pathway promotes translocation of Mdm2 from the cytoplasm to the nucleus. Proc. Natl. Acad. Sci. 98: 11598.

Nowak M., Koster C., and Hammerschmidt M. 2005. Perp is required for tissue-specific cell survival during zebrafish development. Cell Death Differ. 12: 52.

Ogawara Y., Kishishita S., Obata T., Isazawa Y., Suzuki T., Tanaka K., Masuyama N., and Gotoh Y. 2002. Akt enhances Mdm2-mediated ubiquitination and degradation of p53. J. Biol. Chem. 277: 21843.

Sachidanandam R., Weissman D., Schmidt S.C., Kakol J.M., Stein L.D., Marth G., Sherry S., Mullikin J.C., Mortimore B.J., Willey D.L., Hunt S.E., Cole C.G., Coggill P.C., Rice C.M., Ning Z., Rogers J., Bentley D.R., Kwok P.Y., Mardis E.R., Yeh R.T., Schultz B., Cook L., Davenport R., Dante M., and Fulton L., et al. (International SNP Map Working Group). 2001. A map of human genome sequence variation containing 1.42 million single nucleotide polymorphisms. Nature 409: 928.

Soussi T. and Beroud C. 2001. Assessing TP53 status in human tumours to evaluate clinical outcome. Nat. Rev. Cancer 1: 233.

Venter J.C., Adams M.D., Myers E.W., Li P.W., Mural R.J., Sutton G.G., Smith H.O., Yandell M., Evans C.A., Holt R.A., Gocayne J.D., Amanatides P., Ballew R.M., Huson D.H., Wortman J.R., Zhang Q., Kodira C.D., Zheng X.H., Chen L., Skupski M., Subramanian G., Thomas P.D., Zhang J., Gabor Miklos G.L., and Nelson C., et al. 2001. The sequence of the human genome. Science 291: 1304.

Vogelstein B., Lane D., and Levine A.J. 2000. Surfing the p53 network. Nature 408: 307.

Zhou B.P., Liao Y., Xia W., Zou Y., Spohn B., and Hung M.C. 2001. HER-2/neu induces p53 ubiquitination via Akt-mediated MDM2 phosphorylation. Nat. Cell Biol. 3: 973. 


\section{$\$_{\text {CSH\& }}^{\infty} \mathrm{Cold}$ Spring Harbor Symposia SYMPOSIA on Quantitative Biology}

\section{Single-Nucleotide Polymorphisms in the p53 Pathway}

S.L. HARRIS, G. GIL, W. HU, et al.

Cold Spring Harb Symp Quant Biol 2005 70: 111-119

Access the most recent version at doi:10.1101/sqb.2005.70.024

References This article cites 31 articles, 8 of which can be accessed free at: http://symposium.cshlp.org/content/70/111.full.html\#ref-list-1

\section{License}

Email Alerting Receive free email alerts when new articles cite this article - sign up in Service the box at the top right corner of the article or click here. 